\title{
Optimization of a sparse grid-based data mining kernel for architectures using AVX-512
}

Paul-Cristian Sârbu, Hans-Joachim Bungartz

Technical University of Munich

Department of Informatics

Chair of Scientific Computing

$1^{\text {st }}$ HPML Workshop

September 2018, Lyon, France

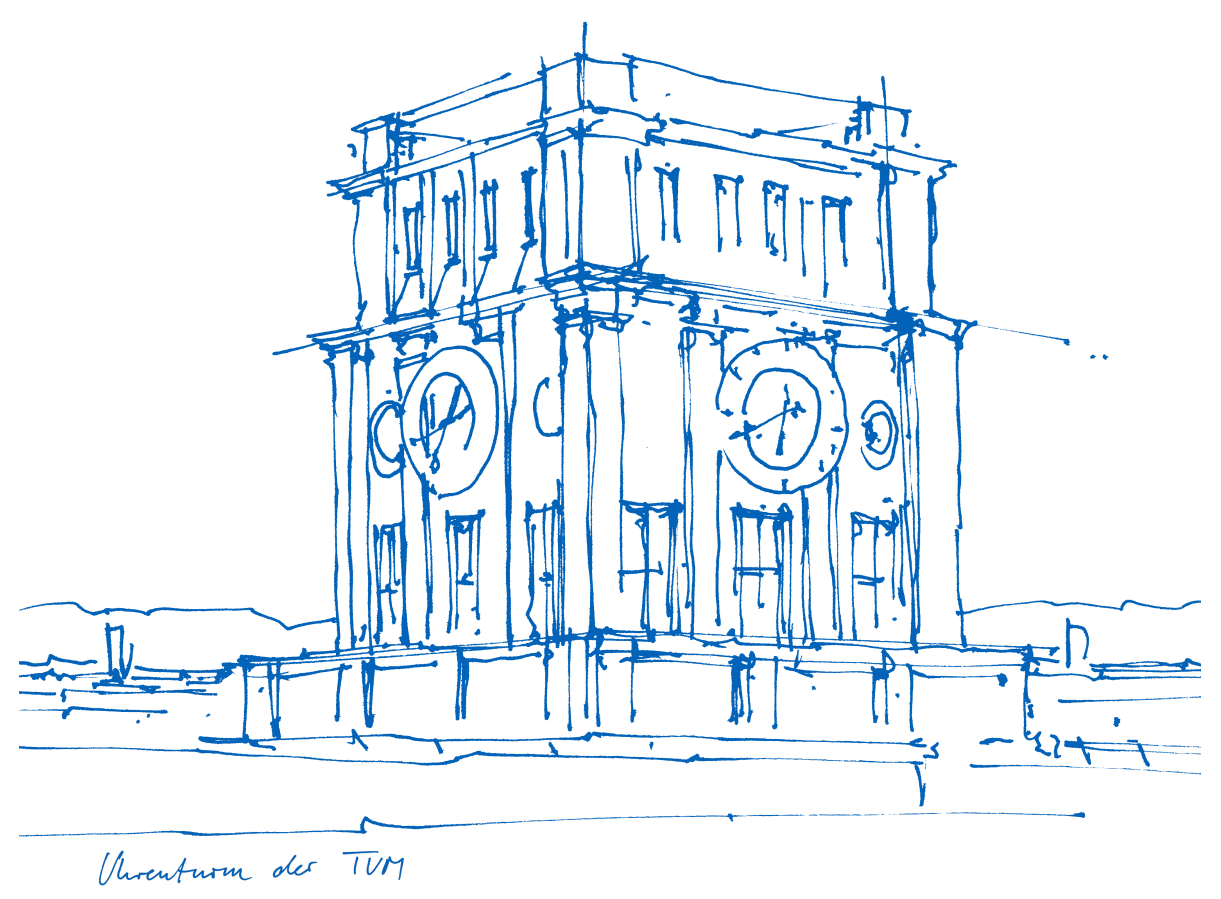




\section{Motivation}

Intel $\circledast$ Parallel Computing Center (IPCC) at Leibniz Supercomputing Center (LRZ) ${ }^{1}$

\section{GADGET SeisSol \\ Is1-mardin SG++}

- Updating legacy code for AVX-512

- Studying influence of hardware specific features $\rightarrow$ Knights Landing

- Performance gains relative to Haswell implementation 


\section{Data mining with Sparse Grids}

$$
\hat{f}_{N}(\vec{x})=\sum_{j=1}^{N} u_{j} \varphi_{j}(\vec{x})
$$

$\left(M \lambda I+B B^{T}\right) \vec{u}=B \vec{y}, b_{j, i}=\varphi_{j}\left(\vec{x}_{i}\right) \quad \rightarrow$ solve with

Conjugate Gradient

$$
M=\text { dataset size } \quad \lambda=\text { regularization parameter }
$$

- Optimizing two matrix-vector operations

$$
\text { mult : } \quad \vec{v}=B^{T} \vec{u} \quad \text { mult } T: \quad \vec{w}=B \vec{v}
$$




\section{Legacy implementation}

3 loops, iterative scheme, manual vectorization, optimized for AVX2

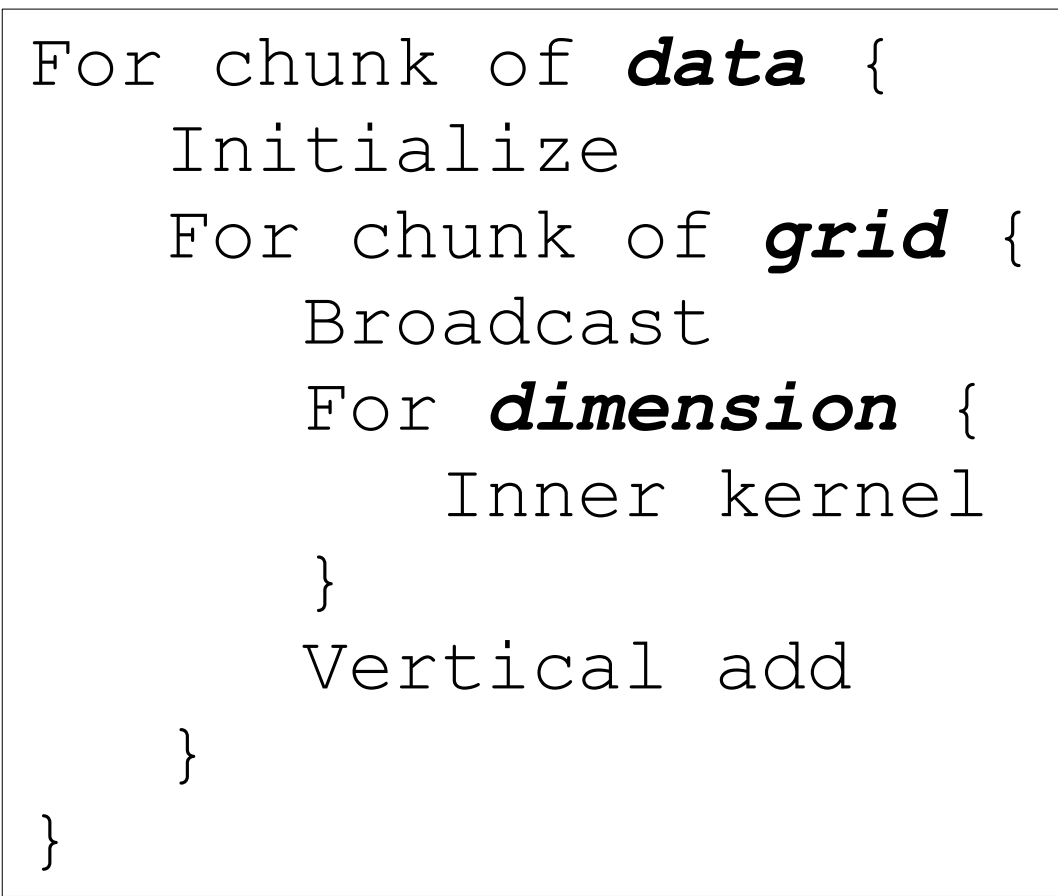

mult

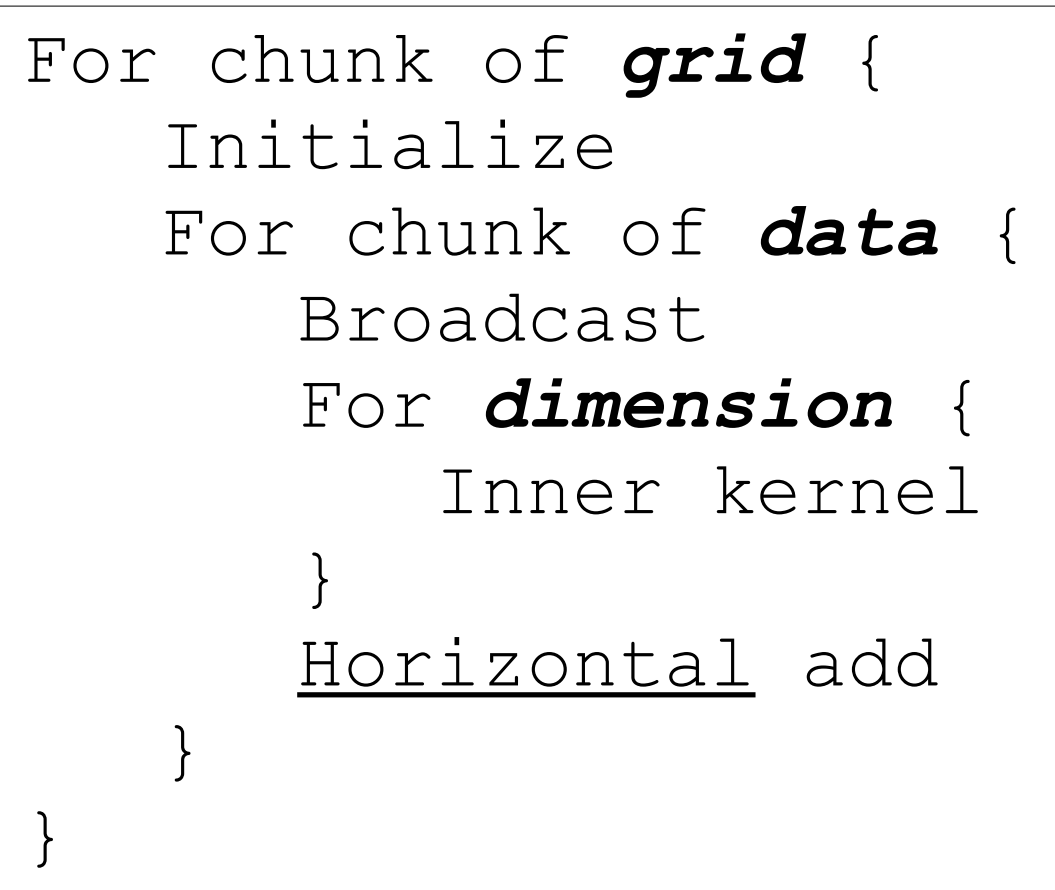

multT 

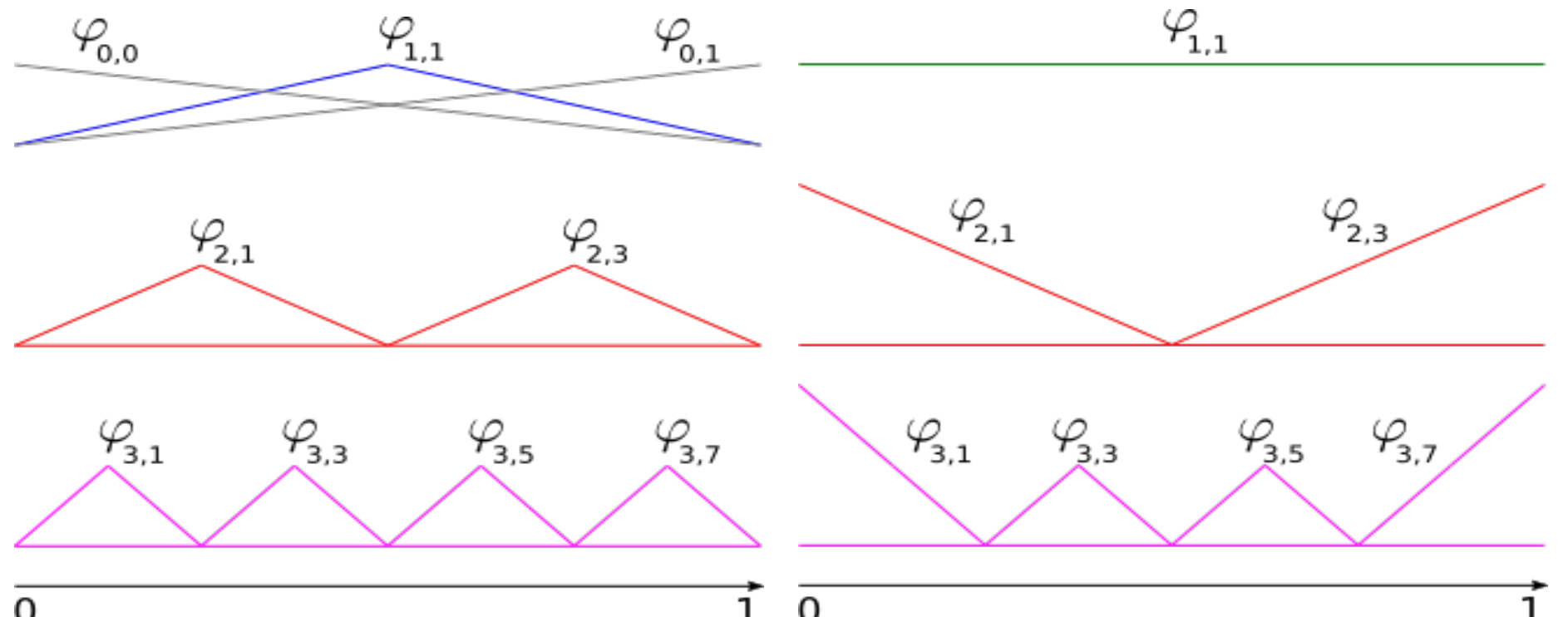

0

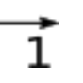

\begin{tabular}{ccccc} 
Basis & $\begin{array}{c}\text { Masking } \\
\text { needed }\end{array}$ & $\begin{array}{c}\text { Peak } \\
\text { performance }\end{array}$ & Grid points & $\begin{array}{c}\text { Time to } \\
\text { solution }\end{array}$ \\
\hline linear & No & \\
modlinear & Yes &
\end{tabular}


Hardware specifications - Knights Landing

- CooLMUC-3 cluster at LRZ1

148 nodes of 64 -cored Xeon

Phi 7210-F @ 1.30 Ghz,

Turbo Mode ON

- Why KNL?

- AVX-512 instruction set

- Level 2 cache (L2) format $\rightarrow$ bidirectional 2D mesh

- 96GB DDR4 + 16GB MCDRAM

- Clustering and memory modes
- CooLMUC-2 cluster at LRZ1

28-core Xeon E5-2697 v3 (Haswell) nodes @ 2.60 Ghz, Turbo Mode ON

$\Delta$

baseline architecture 


\section{Optimization results}

- Intrinsics adaptation

- embedded broadcasts, but no AVX-512DQ $\rightarrow$ Skylake

- OpenMP scheduling study

- dynamic better for few cores, static still better for whole node

- Chunk size study $\rightarrow 2 x$ SIMD registers available

\begin{tabular}{|c|c|c|c|c|c|}
\hline & $\begin{array}{c}\text { Grid } \\
\text { level }\end{array}$ & $\begin{array}{c}\text { Grid } \\
\text { index }\end{array}$ & $\begin{array}{c}\text { Result } \\
\text { vectors }\end{array}$ & $\begin{array}{c}\text { Data } \\
\text { vectors }\end{array}$ & Buffers \\
\hline AVX2 & 1 & 1 & 6 & 6 & 2 \\
\hline AVX-512 & 1 & 1 & 1312 & 1312 & $z 4$ \\
\hline
\end{tabular}




\section{Optimization results}

- 5D binary classification problem, chessboard dataset, up to $2^{28}$ data points ( $\approx 20 \mathrm{~GB}$ )

- Thread-level runs
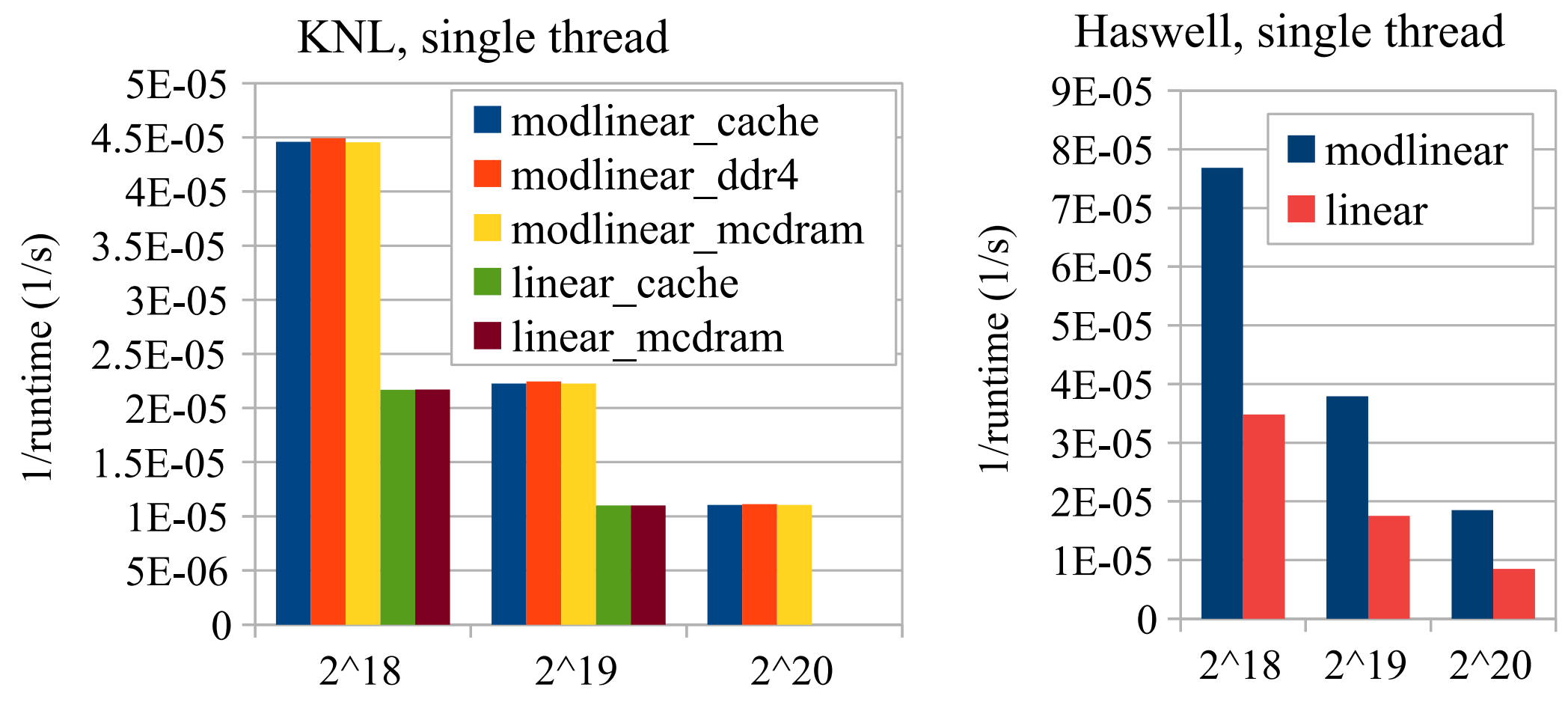


\section{Optimization results}

- Core-level runs
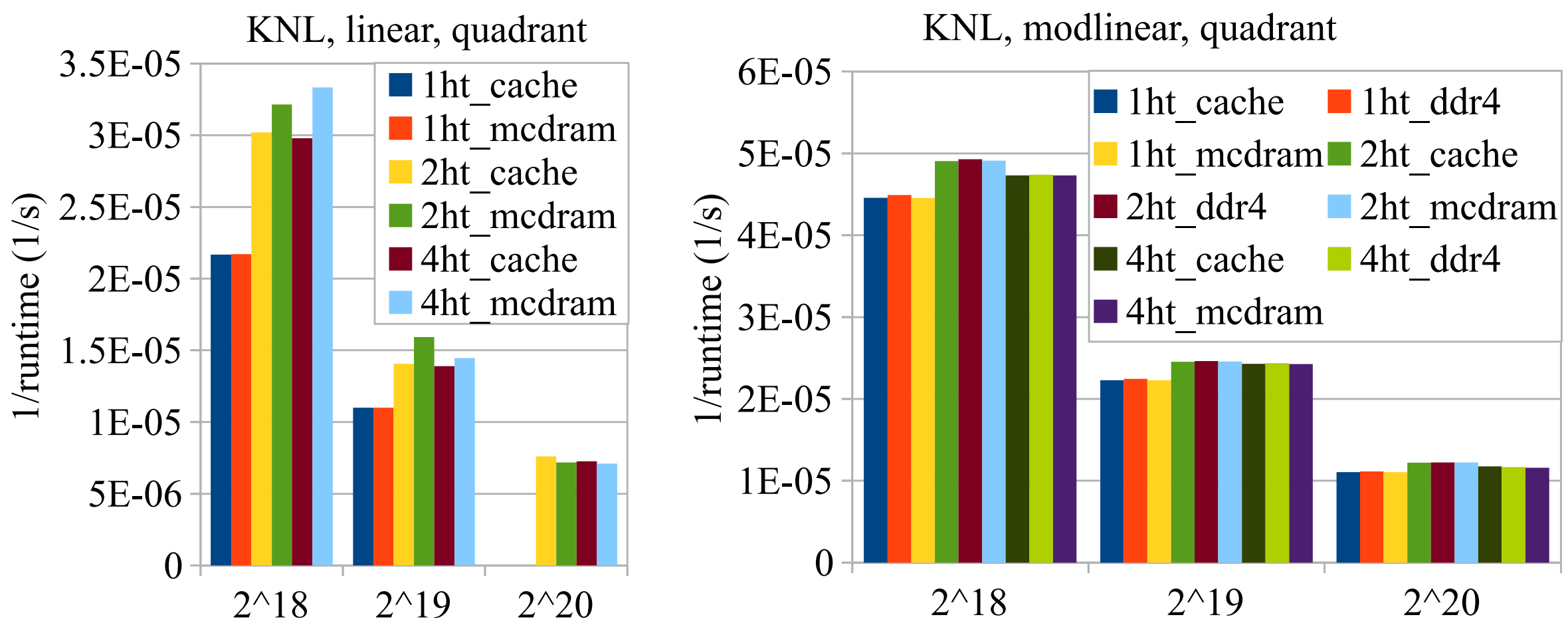


\section{Optimization results}

- Node-level runs

- best configuration: 63 cores $\times 2$ hyperthreads, pure OpenMP, quadrant mode

$>97 \%$ parallel efficiency

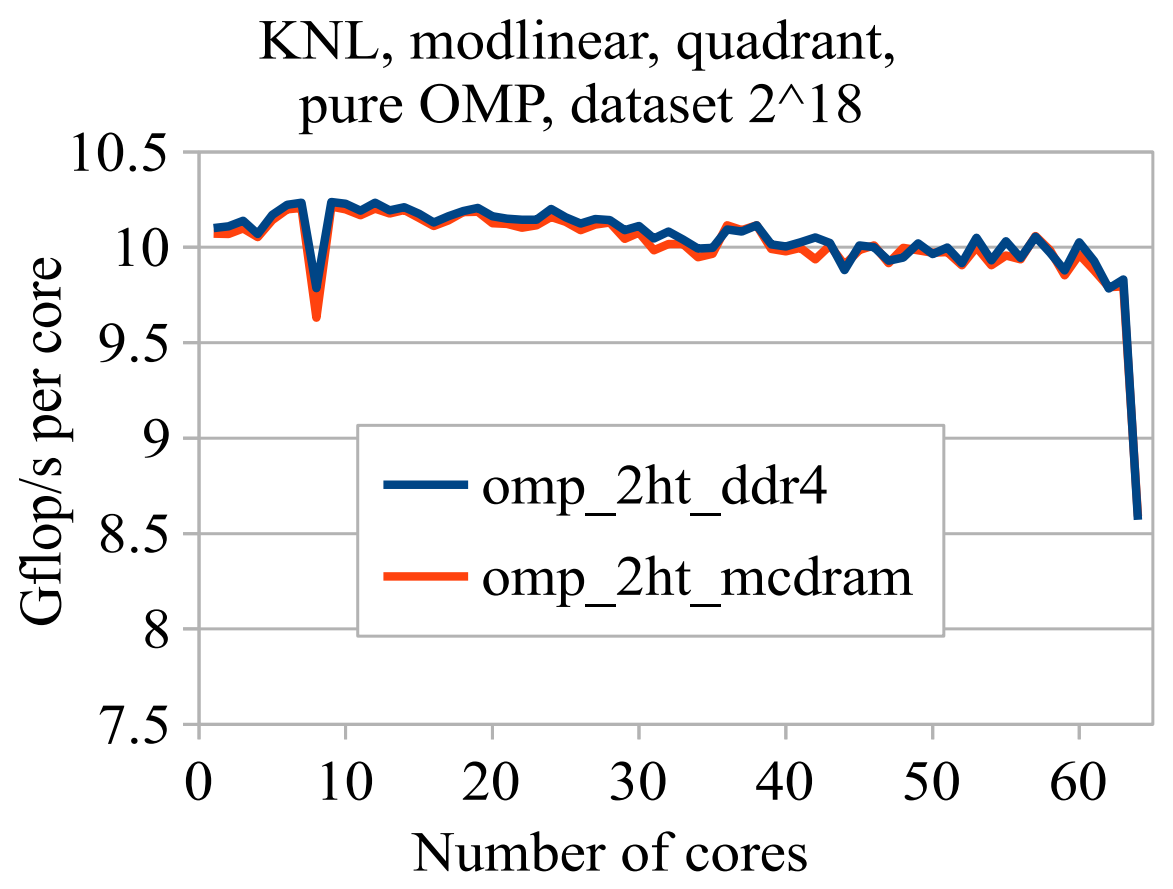

KNL, modlinear, pure MPI, quadrant,

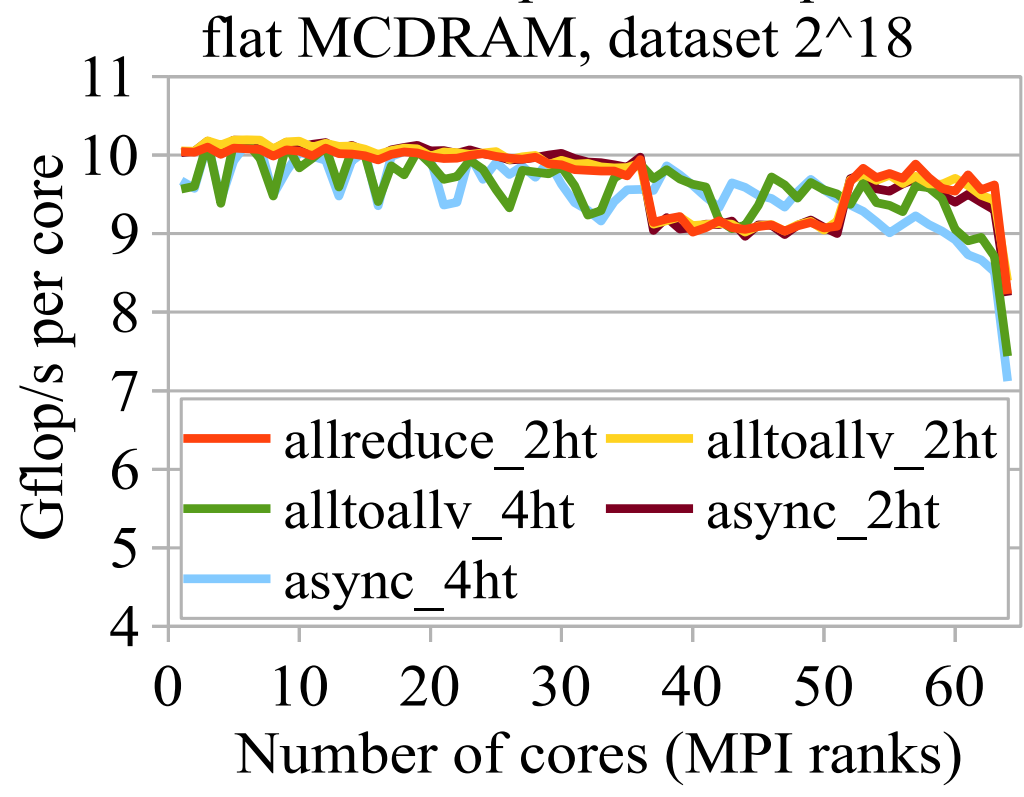




\section{Optimization results}

- Node-level runs

- 1.63 - 1.7x speedup versus Haswell

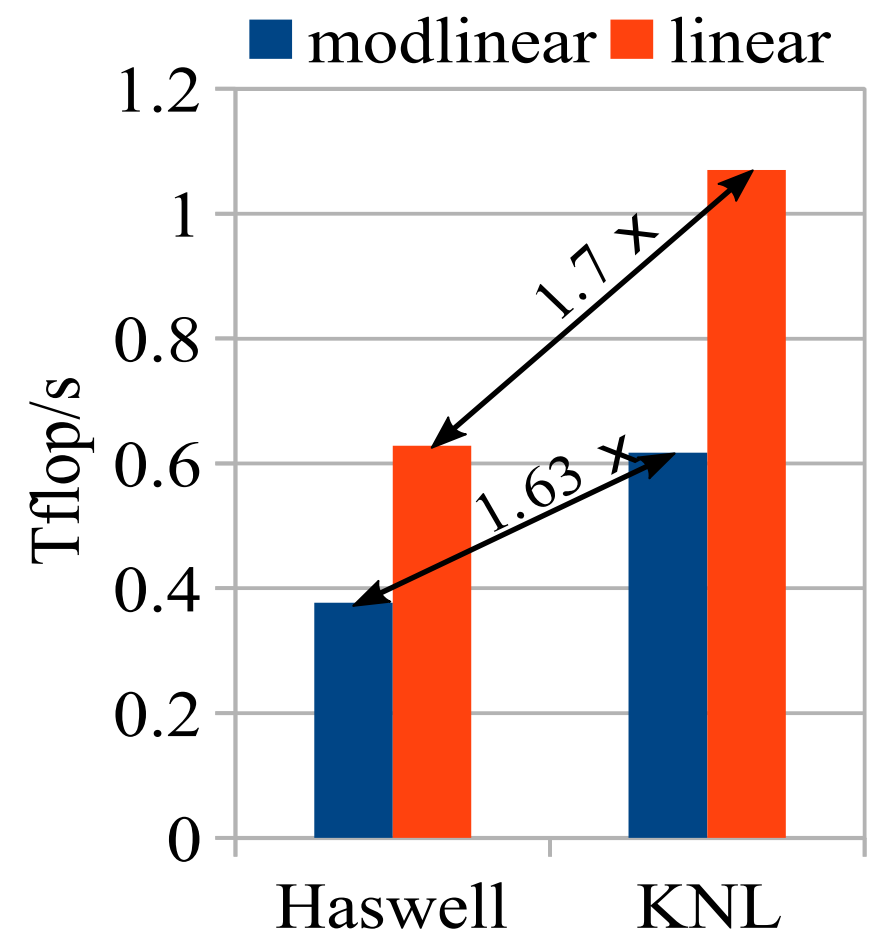




\section{Optimization results}

- Cluster-level runs at MCDRAM size limit

KNL, 2HT/core, 126 omp threads/core, MPI Allreduce, quadrant

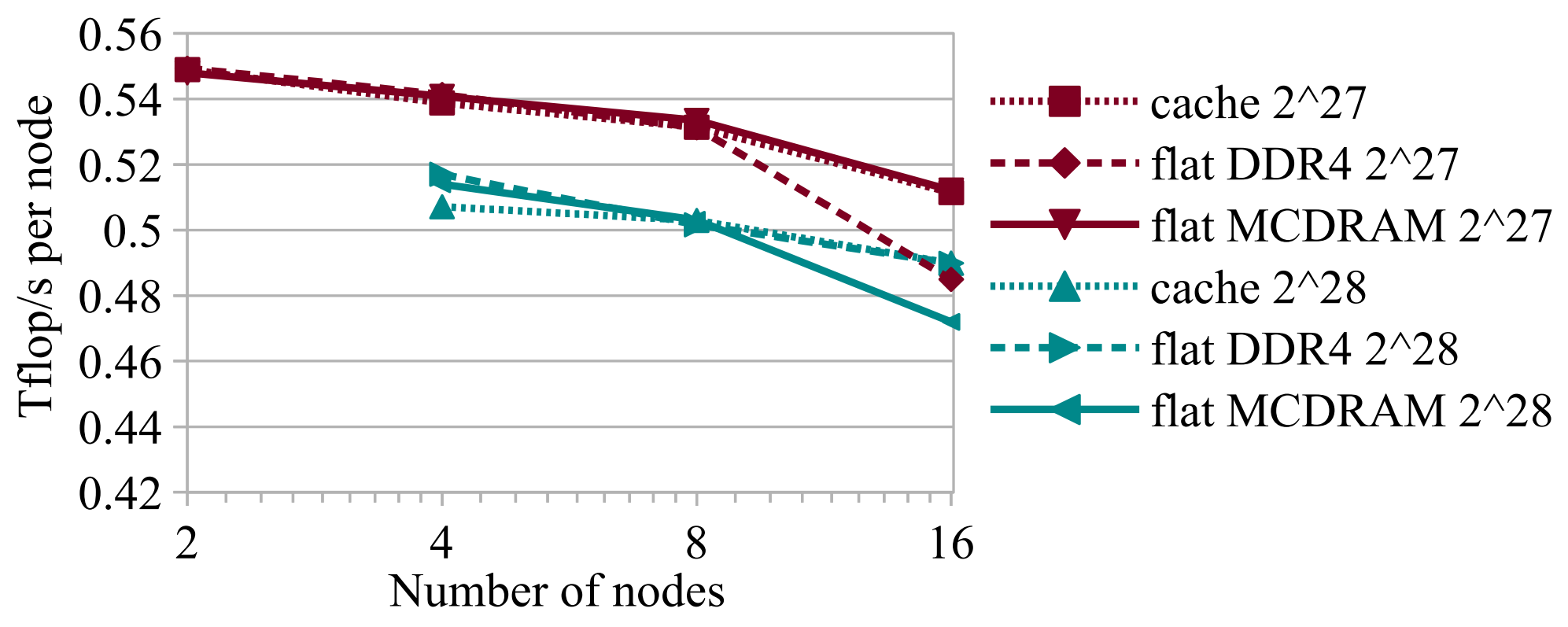

$<16 G B \quad$ flat MCDRAM mode

>16GB cache or flat DDR4 mode 


\section{Conclusions}

- Successful node-level optimization on KNL

- Considerable speedup versus Haswell

- Good behavior at the HBM size limit

\section{Outlook}

- Cluster-level runs (close to DDR4 size limit) on larger machines

- Code ready for Skylake runs 


\section{Conclusions}

- Successful node-level optimization on KNL

- Considerable speedup versus Haswell

- Good behavior at the HBM size limit

\section{Thank you for your attention!}

Contact: sarbu@in.tum.de 


\section{References}

- H.-J. Bungartz and M. Griebel - „Sparse grids“, Acta Numerica, vol. 13, pp. 147269, 2004.

- D. Pflüger - „Spatially Adaptive Sparse Grids for Higher-Dimensional Problems“, Verlag Dr. Hut, München, 2010. ISBN 9-783-868-53555-6

- D. Pflüger, B. Peherstorfer, and H.-J. Bungartz - „Spatially adaptive sparse grids for high-dimensional data-driven problems“, Journal of Complexity, Volume 26, Issue 5, 2010

- Alexander Heinecke - „Boosting Scientific Computing Applications through Leveraging Data Parallel Architectures“, Verlag Dr. Hut, pp. 1-231, 2014. ISBN 978-3-8439-1408-6 


\section{Hardware specifications - Knights Landing - extra}

\section{Clustering modes}

- quadrant

- KNL as symmetric multi-processor

- software transparent

- low L2 miss latencies

- sub-NUMA clustering

- heavily reliant on non-uniform memory access model

- boost for memory bound codes

- all-to-all

- default debug mode

\section{Memory modes}

- cache

- MCDRAM as level 3 cache

- software transparent

- expensive L3 misses

- $\underline{\text { flat }}$

- straight-forward address mapping

- memory pinning required

- reduced data access time

- hybrid

- mixed strategy; fine control needed 